Revue d'histoire de l'Amérique française

ZAS REVUE D.HISTOIRE DE L'AMÉRIQUE FRANÇAISE

\title{
Les Canadiens français et l'établissement de la Confédération
}

\section{Lionel Groulx}

Volume 21, numéro 3a, 1967

Cent ans d'histoire 1867-1967

URI : https://id.erudit.org/iderudit/302718ar

DOI : https://doi.org/10.7202/302718ar

Aller au sommaire du numéro

Éditeur(s)

Institut d'histoire de l'Amérique française

ISSN

0035-2357 (imprimé)

1492-1383 (numérique)

Découvrir la revue

Citer cet article

Groulx, L. (1967). Les Canadiens français et l'établissement de la Confédération. Revue d'histoire de l'Amérique française, 21(3a), 677-694.

https://doi.org/10.7202/302718ar d'utilisation que vous pouvez consulter en ligne.

https://apropos.erudit.org/fr/usagers/politique-dutilisation/ 


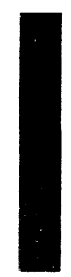

Lionel Groulx

\section{LES CANADIENS FRANÇAIS}

E T

L'ÉTABLISSEMENT

DE LA CONFÉDÉRATION 
Note de la direction:

Nous avons voulu publier, à la fin de ce numéro deux articles du directeur-fondateur de la Revue d'Histoire de l'Amérique Française. On y verra comment, après cinquante ans, puis soixante ans de sa durée, il jugeait l'Acte de 1867. 


\section{LES CANADIENS FRANÇAIS ET L'ÉTABLISSEMENT. DE LA CONFÉDÉRATION*}

De nulle autre province autant que du Bas-Canada n'a dépendu l'établissement de la Confédération. Il fallut compter tout d'abord avec son importance numérique. Au recensement de 1861, il figure avec une population de 1,111,566 âmes. C'est plus que la population réunie des trois provinces du Golfe, plus que le tiers de la population totale du futur État. Aucune fédération n'était possible contre la volonté de ce million d'âmes.

Ce groupe humain peut disposer, en outre, de forces politiques imposantes. Dans le parlement des Canadas-Unis, il est la première puissance, le groupe le moins friable, celui qui, par sa cohésion, vient de faire échouer la politique anglicisatrice de l'Union, et, plus que toute autre cause, a déterminé, sous Bagot puis sous Elgin, l'émancipation coloniale. Pendant que le groupe parlementaire du Haut-Carada n'a de constance que dans sa versatilité, se compose et se décompose selon le caprice de chefs éphémères, le parti de LaFontaine, de Morin, puis de Taché, traite de haut avec les partis et les factions, s'allie tour à tour aux uns et aux autres, sans jamais cesser d'être lui-même, identique dans son principe et ses aspirations. La petite fraction libérale-démocrate a pu s'organiser sur ses flancs; elle l'affaiblit sans en faire une faiblesse. Par surcroît, à l'heure de 1864, le groupe canadien a pour chef un véritable meneur d'hommes. Dépourvu du magnétisme de Papineau, inférieur de caractère à LaFontaine et à Morin, Georges-Étienne Cartier exerce pourtant sur son parti et ses compatriotes, un empire égalé par aucun des chefs, ses prédécesseurs. Plus qu'eux tous il détient les qualités maîtresses du commandement. Il domine par un ascendant impérieux, une personnalité volontaire, nerveuse, presque despotique, qui sait comme l'on s'impose à un peuple de

* Extrait de: Les Canadiens français et la Confédération canadienne (Montréal, Bibliothèque de l'Action française, 1927), 1-21. "Enquête de l'Action française, mai-juin 1927". 
traditions monarchiques. Ce n'est pas que sa race soit prête à vouloir tout ce qu'il voudra; mais elle ne voudra rien qu'il n'ait voulu. Sur ce point tous les contemporains, tous les historiens tombent d'accord: un seul homme, George-Etienne Cartier, pouvait faire agréer à sa province les graves changements politiques de 1867, comme un seul mot de ce même homme pouvait faire s'arc-bouter les siens dans une invincible opposition.

Le Bas-Canada peut alors tenir la dragée haute, par une autre raison décisive et qui est sa situation géographique. Parce que trop simple, sans doute, les historiens ont trop négligé cette donnée de premier ordre. Pour les bâtisseurs de 1864, le BasCanada ne pouvait être que l'arche maîtresse de la construction ou le vide infranchissable. Et non seulement parce qu'avec son havre de Montréal et sa longue part navigable du Saint-Laurent, il constitue entre les provinces l'indispensable route d'échanges et d'union, la suprême artère vitale; mais plus simplement parce qu'il effectue entre les Etats de la mer et ceux du centre, la contiguïté du territoire. Déjà l'on trouvait extravagant et arbitraire de souder politiquement des provinces séparées par les espaces incultes du bas du fleuve. Quel ingénieux artifice eût cimenté une alliance par-dessus une solution de continuité de 300 milles ? Quelle arche gigantesque eût pu couvrir ce vide immense! Le cas est de force majeure en politique et en histoire. Sans le Bas-Canada, une fédération des provinces maritimes se pouvait constituer; nullement une confédération canadienne. Le Haut-Canada n'avait devant lui que ce triple choix: ou son isolement à l'autre bout du pays, ou une fédération bipartite avec le Bas-Canada, ou la prolongation du régime de 1840, accouplement contre nature que chaque jour rendait plus intolérable.

La confédération canadienne dépend, au premier chef, du Bas-Canada; il est aussi de toutes les provinces, celle qui y prend le plus de risques. Quels que fussent ses griefs contre le régime de 1841, ils ne dépassaient point ceux du Haut-Canada. "Nous siégons ici aujourd'hui et cherchons à l'amiable un remède à des maux constitutionnels et à des injustices dont se plaignent les 
vaincus?" demandait George Brown en 1865. "Non, répondait-il, mais dont se plaignent les conquérants!" 1 Puis, l'on ne saurait trop le rappeler: le système fédératif, simple "faute de mieux", ne représente nullement pour les peuples l'état politique parfait; l'état parfait, c'est proprement la libre disposition de soi-même dans la pleine indépendance. De là les principes et les soucis qui président à l'avènement d'une fédération. Les petits peuples se fédèrent pour rendre plus stable leur condition politique et nationale, non pour en accroître les périls. Ils prennent une assurance; non pas de plus grands risques. John A. MacDonald, dans son discours d'Halifax, marquait nettement en 1864 ce caractère essentiel de toute fédération politique:

Dans la discussion d'une union coloniale, nous devons
considérer ce qui est désirable et pratique; nous
devons consulter les préjugés et les aspirations des
parties ... J'espère que nous pourrons mettre au
jour une constitution qui comportera un gouverne-
ment central fort, en état d'offrir un front puissant
contre l'ennemi, quel qu'il soit, et qui, en même
temps, préservera l'iclentité de chaque province et
protégera toutes les aspirations particulières. Et si
nous ne pouvons accomplir cette ouvre politique, il
nous sera impossible d'atteindre les fins que nous
avons maintenant en vue.

Quels biens particuliers, quelles aspirations le Bas-Canada aurait-il à placer sous la protection de l'État fédéral? Le HautCanada n'a besoin de se préoccuper que d'intérêts politiques et matériels. Tout au plus veille-t-il à son système scolaire que, jalousement, il estime inaltérable. Préoccupations de même ordre dans les colonies du Golfe qui, plus faibles que le Haut-Canada, trouvent à craindre néanmoins pour un certain particularisme politique et social. Dans le seul Canada français, les inquiétudes prennent un caractère en quelque sorte transcendant. Sa condition est unique. Dans ce duel de civilisation qui se poursuit ici

1 Débats parlementaires sur la question de la Confédération des provinces de l'Amérique britannique du Nord - $3^{\mathrm{e}}$ Session, $8^{\mathrm{e}}$ Parlement Provincial du Canada - Imprimé par ordre de la Législature (Québec, 1865), 84.

2 The Union of the British Provinces... (Charlottetown, 1865), 44. 
depuis 1760 , il ne peut appréhender qu'une nouvelle péripétie plus étreignante que les autres. Après quatre-vingts ans d'isolement relatif où il avait pu soutenir la défense de son âme contre une infime minorité anglaise et en champ clos, voici qu'en 1840, le champ de bataille soudainement agrandi, il avait dû mêler sa vie à toute une province et à tout un peuple britanniques. En 1864, le champ de bataille s'élargissait jusqu'à la moitié du continent; et le Bas-Canada aurait à jouer sa destinée contre quatre provinces au lieu d'une. Et ce ne sont pas seulement des intérêts politiques et économiques, un particularisme social qu'il met au jeu; mais des intérêts religieux et moraux: tout son être national et toute sa foi. Fils de l'Église, il ne peut oublier qu'il a charge d'âmes et qu'il lui appartient de prendre des garanties, non seulement pour lui-même, mais pour toutes les minorités catholiques du futur État. Pour un peuple qui a la foi du Christ, rien n'égale ces responsabilités.

Aussi bien est-ce un double contrat que le Canada français doit élaborer et signer avec ses partenaires de la fédération: un contrat politique qui réservera toute son autonomie de province, délimitera soigneusement juridictions fédérales et juridictions provinciales; mais aussi un contrat de caractère national et religieux qui, avec les droits de la nationalité canadienne-française, fixera la condition des minorités religieuses dans les provinces. La nature même du contrat fédératif, le Canada français seul ou presque seul la déterminera. Le Haut-Canada, c'est bien connu, eut préféré la simple union législative. Dans les provinces maritimes, l'opposition à cette forme d'État n'a rien d'universel, ni d'irréductible. ${ }^{3}$ Si l'alliance de 1867 devint un pacte fédératif plutôt qu'une fusion de provinces, ce fut chose exigée tout d'abord par le Canada français.

3 "Je sais que des membres de cette Chambre, et nombre de personnes du Haut-Canada et des provinces maritimes pensent qu'une union législative serait plus avantageuse qu'une Confédération." - Discours de G.-E. Cartier, Débats parlementaires sur la question de la Confédération, 1865. Voir aussi Joseph Pope, ed., Confederation: being a series of Hithero unpublished documents bearing on the British North America Act (Toronto, $1895), 59,60-61,82,84-95$, les déclarations fort significatives de $M M$. Fisher, Whelan et Tupper. 
Mais les textes législatifs auraient-ils puissance de tranquilliser un peuple à qui les hasards de l'histoire ne laissaient guère d'illusions sur l'intangibilité des textes les plus sacrés? Comment se libérer d'inquiétude lorsqu'au milieu même des débats, les deux races s'affrontaient en des attitudes et des sentiments si opposés? D'un côté la volonté de faire au faible une condition privilégiée, le désir de la justice jusqu'à la générosité, de la conciliation jusqu'à l'imprudence; de l'autre la résolution opiniâtre de faire sentir au faible la supériorité du fort, de courber les minorités catholiques et françaises sous des textes rigides et mesquins. Loin de s'élever jusqu'aux conceptions des grands fondateurs d'État et d'apercevoir dans la diversité des races et le maintien de leurs énergies créatrices, un moyen de stimuler et de fortifier le génie national, tout un élément anglosaxon, le plus dominateur et le plus écouté, ne se préoccupait que d'apposer au bas du contrat bilatéral, la griffe arrogante du lion, n'admettant d'associé qu'à titre de serf. Quelle lumière crue le débat scolaire de 1866 devait projeter sur ce troublant état d'esprit! Traduisait-il autre chose que l'inquiétude collective, Antoine-Aimé Dorion, lorsqu'au cours des débats parlementaires de 1865, il s'écriait:

Je sais que la population protestante du Bas-Canada craint que, même avee les pouvoirs restreints laissés aux gouvernements locaux, leurs droits ne soient pas protégés. Alors, comment peut-on espérer que le Bas-Canada puisse avoir une grande confiance dans le gouvernement général, qui aura des pouvoirs si immenses sur les destinées de leur section? L'expérience démontre que les majorités sont toujours agressives et portées à être tyranniques, et il n'en peut être autrement dans ce cas-ci. ${ }^{4}$

Quel écho ne trouvait pas dans la conscience populaire cet avertissement de M. Henri.-E. Taschereau, député conservateur de la Beauce et l'un des rares de son parti qui, sur ce point, se séparèrent de leurs chefs :

Je ne saurais dire que nos descendants nous seront reconnaissants pour leur avoir facilité les moyens de

4 Débats parlementaires sur la question de la Confédération... 269. 
faire partie de l'immense empire des provinces anglaises de l'Amérique du Nord. Au contraire, on s'apercevra bientôt que cette confédération est la ruine de notre nationalité dans le Bas-Canada, et le jour où l'on votera cette constitution, on donnera le coup de mort à notre nationalité qui commençait à prendre racine sur cette terre de l'Amérique Britannique du Nord.

Alors s'engagea une lutte que nulle autre, dans l'histoire du Bas-Canada, n'avait égalée en violence. À l'âpreté des querelles de parti vinrent se joindre les passions de fond qu'éveille dans l'âme populaire la conscience des périls suprêmes. Pour la première fois, et sur un enjeu où il y allait de la vie même de la nationalité, les Canadiens français se divisaient en deux camps. Situation pleine de tragique pour un petit peuple dont l'histoire prend en cela même sa grandeur qu'il a toujours tenu son âme et sa survivance pour une grande chose. Etre ou n'être pas fédéraliste, c'était trahir ou ne pas trahir sa race et tous ses espoirs. ${ }^{5}$ Engagée par la jeunesse, dès le lendemain de la Conférence de Charlottetown, la résistance au projet des "Pères" rebondissait avec une force nouvelle, après la Conférence de Québec, par l'énergique manifeste d'Antoine-Aimé Dorion et par les dénonciations de l'Union nationale, groupe où figuraient des jeunes conservateurs en rupture avec leur parti, tel que L.-O. David. Les antifédéralistes ne se recrutent point, en effet, dans la seule faction libérale-démocrate, discréditée d'avance par son américanisme et ses idées irréligieuses. "Nous n'ignorons pas, écrit l'évêque de Saint-Hyacinthe, qu'il y a des hommes pleins de bonne foi et de loyauté, qui redoutent la Confédération, parce que dans quelques-uns de ses détails, elle leur apparaît comme une menace de ruine pour toute influence française et catholique." ${ }^{\circ}$ D'ailleurs, à son assemblée générale aux Trois-Rivières, en l'automne de 1864, l'épiscopat du Bas-Canada décide de laisser

5 Dans sa lettre pastorale du 8 juin 1867, l'évêque des Trois-Rivières signalait pour le déplorer le caractère particulièrement violent de ce débat politique: "C'est avec peine que nous avons vu la violence avec laquelle quelques-uns de nos compatriotes se sont élevés contre le projet de Confédération."

- Lettre pastorale concernant l'inauguration du Gouvernement fédéral. 
M. Clerk, rédacteur au journal catholique le True Witness, discuter les principes de la Confédération projetée, pourvu qu'il respecte le ministère. ${ }^{7}$

Le soulèvement des esprits devient tel qu'après avoir laissé promettre par ses journaux une consultation électorale sur son projet de fédération, le ministère canadien prend peur et juge prudent de n'en rien faire. Savamment il manœuvre pour éviter le verdict populaire, bien convaincu qu'un simple incident, la moindre erreur de tactique, peut tout compromettre. C'est John A. MacDonald qui écrivait à Leonard Tilley le 8 octobre 1866:

Eussions-nous convoqué les Chambres pendant l'année et avant vos élections, vous en eussiez éprouvé le plus grand embarras et probablement votre défaite s'en fút suivie. Nous aurions été pressés par l'opposition de déclarer si nous adhérions aux résolutions de Québec, oui ou non. Si nous avions répondu dans l'affirmative, vous auriez été défaits, puisque vous n'étiez point en état d'aller devant l'électorat, sous le faix de ces résolutions. Eussions-nous répondu dans la négative et déclaré la question ouverte et les résolutions [de Québec] sujettes à modification, le Bas-Canada se serait levé comme un seul homme, et adieu la Confédération. ${ }^{8}$

Quelle puissance magique apaisa donc ces inquiétudes et ces colères et capta l'adhésion du Bas-Canada au projet des "Pères"?

Tout d'abord les chefs politiques, et plus que tout autre, George-Etienne Cartier dont nous savons tout l'empire. Et non pas tant la personnalité ni le prestige de ces chefs que les assurances prodiguées par eux, avec une sorte de surenchère. Ces assurances, ils ont soin qu'elles s'étendent à tous les grands objets pour lesquels on s'inquiète. En premier lieu les éléments

${ }^{7} \mathrm{M} g r$ Bourget à Mgr de Flca, 21 déc. 1864. Archives de ${ }^{2}$ Archevêché do Montréal, Lettres de Mgr Bourget, vol. XXIII: 262.

8 Joseph Pope, Memoirs of the right honourable Sir John Alexander Macdonald, G.C.B., first prime minister of the Dominion of Canada (2 vol., Ottawa, 1894), I: 306. 
mêmes de la nationalité: la langue et la foi. Si les premiers dispositifs de la Confédération de Québec s'avéraient trop concis pour être rassurants, graduellement, au cours des débats, la volonté d'être juste et prévoyant a fait trouver des formules plus amples et plus précises. Pour les droits officiels de la langue, la rédaction définitive aboutit à l'article 133 qui parut tout prévoir et tout protéger; les droits scolaires des minorités vinrent s'abriter sous le minutieux article 93.

Toutefois ces garanties n'ont chance d'être efficaces que si les éléments de l'âme canadienne-française, placés dans un cadre politique autonome, échappent à l'État unitaire, destructeur des particularismes. Sur ce point encore, Cartier et ses lieutenants multiplient les précautions, prennent des assurances contre un camouflage du fédéralisme. Mieux que des fantômes d'État, les provinces seront de vraies entités politiques, douées d'une ample juridiction. Ả la Conférence de Québec comme à celle de Londres, ce n'est point le vote personnel des délégués qui a fixé la constitution; c'est par province que l'on a voté; et, pour marquer sa dualité ethnique et politique, le Canada a disposé de deux voix. Le préambule même de la nouvelle charte affirme, sans ambages, le maintien du provincialisme: "Considérant que les provinces du Canada, de la Nouvelle-Écosse et du Nouveau-Brunswick ont exprimé le désir de contracter une Union Fédérale... Considérant de plus qu'une telle union aurait l'effet de développer la prospérité des provinces..." Fusionné dans le préambule avec l'Ontario, sous le titre de Canada, voici qu'à l'article 5, le Québec recouvre son entité politique distincte: "Le Canada sera divisé en quatre provinces, dénommées: - Ontario, Québec, NouvelleÉcosse et Nouveau-Brunswick." Que l'on n'oppose point à Cartier ce terme de "nationalité nouvelle" alors en vogue, - on dirait aujourd'hui de "canadianisme tout court" - et qui indiquait une fusion plutôt qu'une fédération de nationalités. Dès ce temps-là, avec son esprit réaliste, l'homme d'État canadien a fort justement démêlé la part du vrai et du faux, du possible et du chimérique, dans ce rêve d'une nouvelle nationalité. Nationalité canadienne, oui ; au sens politique du mot, nullement au sens ethnique. "On a fait objection à notre projet à cause des mots "nouvelle 
nationalité" qui s'y rencontrent, observait Cartier; si nous nous unissons, nous formerons une nationalité politique, indépendante de l'origine nationale et de la religion des individus." Pour plus de clarté, il ajoutait tout aussitôt:

Des hommes ont regretté qu'il y eût diversité de races et ont exprimé l'espoir qu'avec le temps cette diversité disparaîtrait. La fusion des races en une seule est une utopie; c'est une impossibilité.

Maîtresse chez elle, dans le cadre autonome de sa province, la race canadienne-française, d'après Cartier, disposerait aussi dans le cadre fédéral, de suffisantes protections. Sur ce champ de bataille plus périlleux, avec fermeté, il indiquait la seule tactique décisive pour les siens, tactique dont l'abandon nous a valu peut-être une partie de nos défaites:

Quand un leader du Bas-Canada aura soixante-cinq représentants pour l'appuyer, et qu'il pourra disposer d'une majorité des Canadiens-Français et des Anglais du Bas-Canada, ne pensez-vous pas qu'il pourrait renverser le gouvernement si ses collègues s'opposaient à ses recommandations? C'est là notre garantie. Actuellement si l'on me faisait une opposition déraisonnable, j'aurais le remède tout prêt, celui de me retirer et de précipiter par là-même la chute du gouvernement. Il en sera de même dans le gouvernement fédéral.

Sans doute, les discours de Cartier et de ses lieutenants attestent peu de clairvoyance. Telles déclarations du chef conservateur, et telles autres de Langevin et de Cauchon feraient sourire aujourd'hui, n'était le prix qu'elles nous ont coûté. Ces hommes étaient-ils sincères? Nous le croyons. Leur illusion et leur tort - et l'un et l'autre sont grands - furent de trop accorder à la bonne foi de leurs associés politiques, prenant ainsi sur l'avenir des gages téméraires. Où donc les hommes qui venaient de vivre la belliqueuse histoire du dernier quart de siècle prenaient-ils le droit de s'abandonner à pareil optimisme?

Leur excuse, si une excuse peut être invoquée, c'est de n'avoir rien promis que n'aient autorisé les assurances verbales 
des chefs de la majorité. Quoi de plus net, par exemple, que cette déclaration de John A. MacDonald, qualifié plus que tout autre pour dire le sentiment des siens à l'heure de la signature du contrat fédératif:

Les délégués de toutes les provinces ont consenti à ce que l'usage de la langue française formât l'un des principes sur lesquels serait basée la Confédération ...9

Et quelle foi ne pas ajouter à ces déclarations, lorsqu'au parlement impérial, lord Carnarvon les confirmait ainsi de sa haute autorité:

Le Bas-Canada est jaloux et fier à bon droit de ses coutumes et de ses traditions ancestrales; il est attaché à ses institutions particulières et n'entrera dans l'union qu'avec la claire entente qu'il les conservera ... Et c'est avec ces sentiments et à ces conditions que le Bas-Canada consent maintenant à entrer dans cette Confédération.

Puis encore :

L'objet de cet article (art. 93) ... a été de placer toutes les minorités, à quelque religion qu'elles appartiennent, dans une parfaite égalité de situation, que ces minorités soient in esse ou in posse..$^{10}$

Ces paroles des chefs politiques pesèrent d'un lourd poids sur l'opinion canadienne-française. Auraient-elles emporté toute résistance, si des paroles plus augustes n'étaient venues les appuyer? Que l'épiscopat du Bas-Canada ait virtuellement tenu dans ses mains le sort de la Confédération, nul, croyons-nous, n'en saurait disconvenir. L'empressement de Cartier à se prévaloir de l'approbation des évêques, les fureurs des libérauxdémocrates contre les lettres pastorales de 1867 laissent assez voir le cas que l'on fit de l'opinion épiscopale. Les évêques n'avaient nul besoin de jeter du côté de l'opposition, leur in-

${ }^{9}$ Débats parlementaires sur la question de la Confédération, 1865, 943. 10 Parliamentary Debates, 1867,568 et 556. 
fluence morale; leur silence eût suffi pour tuer dans l'œuf le projet des "Pères".

L'attitude épiscopale, disons-le tout de suite, se fera beaucoup plus discrète que celle des chefs politiques. Les évêques du Bas-Canada savent se garder tout d'abord de la ferveur enthousiaste de leurs collègues des provinces maritimes. ${ }^{11} \mathrm{Au}$ fond ils sont inquiets.

Nous comprenons les hésitations et les alarmes même d'un certain nombre de nos compatriotes, dira Mgr Cooke des Trois-Rivières; et ce n'a pas été sans éprouver nous-même une bien vive émotion que nous avons vu la redoutable nécessité d'en venir à l'adoption d'une mesure si grande en elle-même et dans ses conséquences. ${ }^{22}$

Ces inquiétudes, on les ressent surtout à Montréal, dans l'entourage de Mgr Bourget, le grand homme de l'épiscopat canadien. Quand Cartier se vante en Chambre d'avoir pour lui le sentiment des évêques, M. Truteau, vicaire général de Montréal, se hâte de faire cette réserve:

M. Cartier ... aurait dit en pleine Chambre que les plus hautes dignités des églises de chaque diocèse du Bas-Canada étaient en faveur de la Confédération. $\mathrm{Je}$ ne sais ce que les autres Evêques pensent làdessus; mais quant à Monseigneur de Montréal, je suis positif à dire qu'il n'a pas voulu se prononcer jusqu'ici sur cette question. Avant de partir pour Rome, il disait qu'il aimait mieux attendre avant de prendre aucun parti là-dessus. Pour moi dans mon particulier, je sens que le pays est dans une position exceptionnelle. Je suiss bien persuadé que M. Cartier a d'excellentes intentions et qu'il ne voudrait rien faire qui pût préjudicier au bien de l'église. Mais, je vous avouerai que, tous les jours, je demande à Dieu que, si la Confédération a lieu, il n'en résulte aucun désavantage pour le Bas-Canada, et que nous n'ayons jamais le chagrin de voir notre heureux pays persécuté par la partie protestante, comme cela

${ }^{11}$ Abbé Lionel Groulx, La Confédération canadienne, ses origines (Montréal, 1918), 116, 164-65.

12 Lettre pastorale du 8 juin 1867. 
se voit malheureusement en Suisse, où les Cantons protestants persécutent les Cantons catholiques. ${ }^{13}$

A Montréal l'on s'inquiète en particulier pour ce pouvoir de légiférer sur le mariage et le divorce qui serait accordé au parlement fédéral. M. Truteau eût souhaité une ferme opposition des députés catholiques à cette mesure, laissant "à chaque législature particulière le droit de prononcer sur le divorce". "La législature du Bas-Canada, disait-il, paraissant devoir être toujours composée en majorité de membres catholiques ne nous donnerait pas le hideux spectacle que présente le divorce partout où il est autorisé." 14 Tous les évêques du Canada trouvent à s'alarmer pour les droits scolaires des minorités. Quand, à la session de 1866, le projet de loi Langevin paraît ignorer la minorité du Haut-Canada, l'épiscopat, dans une supplique collective, fait savoir au gouverneur général, lord Monck, “qu'en bonne justice tous les droits et privilèges accordés à la minorité protestante du Bas-Canada doivent être également conférés à la minorité catholique dans le Haut-Canada". Quelques jours plus tard, pour appuyer le sentiment populaire, les évêques, sur proposition de $\mathrm{Mgr}$ de Montréal, décident de rendre publique leur supplique à lord Monck.

Ce ne sera qu'après le vote de la constitution fédérative par le parlement impérial et sa publication dans la Gazette officielle du Canada, à la veille des élections de 1867, qu'enfin les évêques du Bas-Canada se décideront à dire leur sentiment sur le nouvel État politique. Les libéraux-démocrates ont furieusement dénoncé cette "ingérence" des autorités religieuses. Etait-ce tactique bien adroite? Puisqu'en dépit des interventions épiscopales, l'opposition décidait de ne point désarmer, le plus habile moyen d'atténuer le coup n'était-ce pas plutôt de montrer, dans ces lettres des évêques, la discrétion calculée, l'extrême sobriété des éloges décernés au contrat fédératif? Vainement l'on y chercherait, en

${ }^{13}$ M. Truteau à M. C.-F. Cazeau, vicaire général de Québec, 20 février 1865, Archives de l'Archevêché de Montréal, Lettres de Mgr Bourget, vol. XXIII : 288-290.

${ }_{14} \mathrm{M}$. Truteau à Mgr Bourget, 3 fév. et 31 mars 1865; M. Truteau à M. Cazeau, 14 février, 20 février, 3 avril, 12 avril 1865, Archives de l'Archevêché de Montréal, Lettres de Mgr Bourget, vol. XXIII. 
effet, un jugement explicite sur la valeur objective de la constitution. Que l'on se donne la peine d'y regarder de près. Pour imposer la charte de 1867 à l'adhésion des fidèles, les évêques ne savent trouver que des raisons extrinsèques: nécessité de cette forme politique pour le développement matériel du pays, urgence d'un palliatif contre l'annexion aux Etats-Unis, sagesse présumée de cette "œuvre d'hommes d'Etat les plus éminents de chacune des provinces", enfin et surtout, après le vote du parlement impérial et la sanction de Sa Majesté, devoir d'obéissance au gouvernement établi. Des garanties victorieuses pour la protection des droits ethniques et religieux, ils se gardent bien d'en montrer. S'ils en aperçoivent, c'est de façon fort indirecte, dans la mesure où le nouvel État politique préservera de l'annexion américaine ou mettra fin à l'intolérable régime de l'Union des Canadas. Leurs illusions se font si modérées que tous, en face de l'avenir, posent, pour condition expresse de salut, l'union morale et politique des Canadiens français. L'un d'eux, l'évêque de Saint-Hyacinthe, qui croit même aux batailles inévitables, tâche à les faire accepter en les montrant salutaires:

Sans doute, que sous la Confédération, écrit-il, notre position exceptionnelle à cause de notre croyance et de nos mœurs, est bien un peu perplexe et grosse de difficultés! Eh bien, nos très chers frères, nous vous le disons avec conviction: tant mieux pour nous qu'il en soit ainsi, puisque pour un peuple aussi bien que pour un individu, une occasion de lutte rencontrée avec courage et énergie, est un moyen assuré de multiplier ses forces et de se faire respecter!

L'attitude du vénérable évêque de Montréal se fait encore plus défiante. $\AA$ Cartier qui lui a fait tenir une copie du North America Bill, Mgr Bourget a déjà répondu par un simple accusé de réception. ${ }^{15}$ Le temps veriu de tracer une ligne de conduite à ceux qui dépendent de lui, l'évêque le fait par une circulaire à son clergé en date du 25 mai 1867, et par une lettre pastorale du 25 juillet de la même année. L'une et l'autre portent comme titre, non comme aux Trois-Rivières, à Saint-Hyacinthe et à Rimouski: "Lettre Pastorale", ou "Mandement à l'occasion de

15 Abbé Lionel Groulx, La Confédération canadienne, 117. 
la nouvelle Constitution...", “concernant l'inauguration du Gouvernement fédéral", ou "au sujet de la proclamation de la Confédération"; mais "circulaire sur la conduite à tenir pendant les prochaines élections", et "Lettre pastorale indiquant des prières publiques à l'occasion des prochaines élections". Contrairement aux autres documents épiscopaux, la circulaire comme la Lettre pastorale de Montréal n'offrent rien non plus d'un plaidoyer en faveur du nouveau régime. Occasionnellement, et parmi quelques principes de morale sociale, se glisse un simple rappel d'obéissance "à tout gouvernement légitimement établi", et, dans la Lettre pastorale, l'exemple de l'Église du Canada vient appuyer le rappel du même principe. ${ }^{16}$

De cette réserve rien ne pourra faire sortir l'évêque de Montréal. La presse libérale-démocrate, friande de tapage, aura beau opposer cette attitude à celle des autres évêques, et ceux-ci solliciter de leur collègue une déclaration plus explicite, il ne fléchira point. A Mgr Larocque qui lui écrit: "En présence d'un si dommageable état de choses, votre Grandeur ne pourrait-elle pas se prononcer, ne devrait-elle pas même le faire, assez ouvertement et publiquement, pour faire cesser le scandale semé par la malice et la mauvaise foi sur la voie des faibles ?", ${ }^{17} \mathrm{Mgr}$ Bourget fait cette réponse significative: "Je crois devoir déclarer, dans la sincérité de mon âme, que je pense avoir dit, dans ma circulaire et ma Lettre pastorale ... tout ce que je pouvais et devais dire sur la Confédération, pour la direction du clergé et l'instruction du peuple." 18

En résumé, les évêques du Bas-Canada imposèrent à leur peuple l'acceptation du régime fédératif, pour des motifs de circonstances et au nom de la doctrine. Par loyauté ils se gardèrent

16 "Vint ensuite la Constitution, puis l'Union des deux Provinces. L'Eglise s'y soumit et prêcha à ses enfants l'obéissance à l'autorité constituée. Aujourd'hui, elle accepte sans réplique le Gouvernement fédéral, parce qu'il émane de la même Autorité." (Mandements, lettres pastorales, circulaires et autres documents publiés dans le diocèse de Montréal depuis son érection (8 vol., Montréal, 1887), V: 240).

$17 \mathrm{Mgr}$ Larocque à Mgr Bourget, 12 oct. 1867, Archives de l'Archevêchó de Montréal, S.-Hyacinthe, Lettres 1864-81.

$18 \mathrm{Mgr}$ Bourget à Mgr Larocque, Archives de l'Archovêché de Montréal, Lettres de Mgr Bourget, vol. XXV: 458-59. 
bien de le faire au nom d'une confiance qu'ils ne partageaient point. Tout au plus, comme Mgr Cooke des Trois-Rivières, se portent-ils garants de la bonne foi des "Pères" canadiens-français: "Nous ne connaissons rien qui puisse autoriser à croire que la Confédération soit un acte de trahison." ${ }^{19}$ Mais entre eux, l'unanimité s'était faite pour rappeler aux fidèles le grave précepte de l'obéissance à l'autorité constituée. Un tel enseignement et de si hauts témoignages, se joignant à l'autorité des chefs politiques, devaient briser toute opposition. ${ }^{20}$ Votée au parlement de 1865 par à peine vingt-sept députés canadiens-français contre vingt-deux, la Confédération, devenue "fait accompli", ralliait, aux élections de 1867, cinquante-trois comtés du Québec sur soixante-cinq.

$\mathrm{Au}$ souvenir de leur rôle décisif dans l'établissement de la Confédération, les Canadiens français sauront-ils se demander, le ler juillet prochain, quel compte leur fut tenu de leur bonne volonté? Nos associés politiques d'aujourd'hui se souviendront-ils que notre acquiescement de 1867 fut accordé à la bonne foi de nos chefs religieux et politiques, lesquels s'appuyaient, à leur tour, sur la bonne foi des "Pères" de la majorité? S'ils eussent prévu tant de persécutions scolaires et de manquements de paroles, croit-on que les évêques du Bas-Canada, par exemple, auraient écrit leurs Lettres pastorales en faveur de la Confédé-

${ }^{19}$ Le simple vote du parlement des Canadas-Unis, puis la ratification de ce premier vote par le parlement impérial, lequel, se défendant de vouloir rien imposer au peuple du Canada, ne prétendit que ratifier l'œuvre des Conférences et des parlements provinciaux, ces votes des deux parlements suffisaient-ils à faire de l'Acte de l'Amérique $d u$ Nord britannique, le "gouvernement établi" ? En d'autres termes les parlements pouvaient-ils, en pays britannique, changer la constitution sans consultation préalable du peuple? Il y a là un problème de Droit naturel dont la discussion nous entraînerait trop loin.

20 "Le clergé catholique se montra tout particulièrement favorable au projet d'union, et cela a dúi exercer une influence considérable pour réaliser l'œuvre d'un Canada confédéré. Sans Cartier et le clergé catholique de Québec, l'union de 1867 n'aurait pas pu s'accomplir." (Sir John Wilson, Wilfrid Laurier and the Liberal Party, cité par John Boyd, Sir George-Etienne Cartier, Baronnet, sa vie et son temps - Histoire politique du Canada de 1814 à 1873. Ouvrage traduit de l'anglais par Sylva Clapin, en commémoration du centième anniversaire de naissance de Sir George-Etienne Cartier (Librairie Beauchemin, Montréal, 1918), 306. 
ration? Nous ne nions point les illusions ni l'imprévoyance naïve des "Pères" canadiens-français. Nous retenons seulement que s'ils n'ont pas pris plus de garanties, ce n'est pas qu'il ne leur ait paru nécessaire d'en prendre; c'est qu'ils n'ont pas cru, qu'entre citoyens d'un même pays, il fût nécessaire d'en prendre davantage.

L'on convient qu'après plus d'un demi-siècle d'existence, la Confédération canadienne reste encore un géant anémique, porteur de maints germes de dissolution. D'autre part, c'est un principe de biologie générale qu'un être organique dépérit et se corrompt dès que les causes qui lui ont donné sa constitution et sa forme ont cessé d'agir. Si la Confédération canadienne ne doit pas être qu'un État artificiel, une façade sur la frontière américaine, il est temps de ne plus contrarier les forces et les principes qui ont présidé à la formation de ce grand corps politique et qui devaient lui fournir la poussée vitale. Tout ce qu'on a tenté depuis soixante ans, et tout ce que l'on tentera dans l'avenir contre la sécurité de la race canadienne-française en ce pays, on l'a tenté et on le tentera contre son intérêt à maintenir la Confédération. Elle n'y est pas entrée pour y mourir, ni même pour s'y laisser entamer; mais pour y vivre, y subsister intègrement. Ce n'est donc pas l'heure de subtiliser ou de rétrécir l'esprit fédéral; il doit d'autant plus se fortifier et se généraliser à travers le Canada que le contact des deux races s'y est plus étendu. La race canadienne-française n'est plus cantonnée dans l'est du pays; malgré les barrières dressées devant elle, elle a exporté des hornmes dans toutes les provinces occidentales, jusqu'aux côtes du Pacifique. Les réactions de ces groupes français aussi bien que celles du Québec actuel contre les dénis de justice et les mesquineries administratives devraient avertir que si jadis l'on put troquer à bon marché notre adhésion au pacte fédératif, la génération d'aujourd'hui n'admet point qu'on ait vendu ses chances de vie, non plus que son droit de vivre dignement.

LIONEL GROULX, ptre 